\title{
Comparación entre el efecto de la dieta cetogénica y la dieta alta en hidratos de carbono en el aumento de la masa y la fuerza muscular
}

\author{
Comparison between the effect of ketogenic diet and high-carbohydrate diet in the increase \\ of muscle mass and strength
}

Fecha de recepción y aceptación: 23 de julio de 2019, 24 de abril de 2020

DOI: $10.46583 /$ nereis_2020.12.476

\author{
M. Borruel Abadía ${ }^{1}$, M. L. Moreno Sancho², S. Carrera Juliá ${ }^{3}$ y E. Drehmer Rieger $^{1^{*}}$ \\ ${ }^{1}$ Departamento de Ciencias de la Salud. Facultad de Ciencias de la Actividad Física y del Deporte. Universidad Católica de Valencia \\ San Vicente Mártir. \\ ${ }^{2}$ Departamento de Ciencias Básicas. Facultad de Ciencias de la Actividad Física y del Deporte. Universidad Católica de Valencia San \\ Vicente Mártir. \\ ${ }^{3}$ Departamento de Nutrición y Dietética, Facultad de Medicina y Odontología, Universidad Católica de Valencia San Vicente Mártir. \\ ${ }^{*}$ Correspondencia: Universidad Católica de Valencia San Vicente Mártir. Facultad de Ciencias de la Actividad Física y del Deporte. \\ Departamento de Ciencias de la Salud. Calle Ramiro de Maeztu, 14. 46900 (Torrente), Valencia. España.E-mail: eraci.drehmer@ucv. \\ es
}

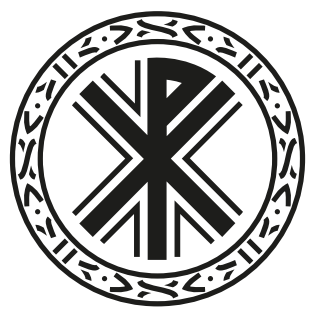

\section{RESUMEN}

La evidencia científica muestra la importancia de la nutrición en la hipertrofia muscular, especialmente con el objetivo de lograr un balance energético positivo entre la ingesta alimentaria y el consumo de energía, siempre y cuando haya una buena ingesta de proteínas. Diversos estudios demuestran que la combinación de ejercicio contra resistencia con un sobreaporte energético produce ganancias en la masa corporal magra. Sin embargo, no está clara la cantidad exacta de todos los macronutrientes. 10 varones con más de 2 años de experiencia en entrenamiento sistemático de la fuerza, fueron divididos en 2 grupos de 5 sujetos, un grupo sometido a dieta cetogénica y el otro a una dieta con alto porcentaje de carbohidratos, y los dos durante 8 semanas. Ambas dietas produjeron un aumento significativo en el porcentaje de masa muscular y en el 1RM de press banca y sentadilla, siendo este aumento más destacado en la dieta rica en carbohidratos. Además, la dieta cetogénica destacó por la disminución significativa del porcentaje de grasa y de peso graso. Por tanto, ambas dietas estarían indicadas para personas que quieren mejorar su masa muscular y fuerza llevando a cabo entrenamiento de hipertrofia. Sin embargo, si se busca más fines estéticos, la dieta cetogénica podría aportar algo más de resultados, y si hay un objetivo más de mejora de la fuerza, sería más recomendable una dieta rica en hidratos de carbono.

PALABRAS CLAVE: dieta cetogénica, dieta hipercalórica, hipertrofia muscular, fuerza, nutrición. 


\begin{abstract}
Scientific evidence shows the importance of nutrition in muscle hypertrophy, especially with the aim of achieving a positive energy balance between food intake and energy consumption, as long as there is always a suitable protein intake. Several studies show that the combination of exercise against resistance with excessive energy consumption produces gains in lean body mass. However, the exact amount of all macronutrients is not clear. 10 men with more than 2 years of experience in systematic strength training were divided into 2 groups of 5 subjects, one group submitted to a ketogenic diet and the other to a diet with a high percentage of carbohydrates, both groups for 8 weeks. Both diets produced a significant increase in muscle mass percentage and $1 \mathrm{MR}$ bench press and squat. This increase was more prominent in the carbohydrate-rich diet. In addition, the ketogenic diet stood out for the significant decrease in fat percentage and fat weight. Therefore, both diets would be indicated for people who want to improve their muscle mass and strength by performing hypertrophy training. However, if the aim is good physical appearance, the ketogenic diet could be more effective, as opposed to strength improvement, to which a diet rich in carbohydrates would be more suitable.
\end{abstract}

KEYWORDS: hypercaloric diet, ketogenic diet, muscle hypertrophy, nutrition, strength.

\title{
INTRODUCCIÓN
}

Cualquier deportista tiene unas necesidades nutricionales basadas en diferentes factores, como la modalidad deportiva, el sexo, la edad, etc., las cuales han de ser cubiertas por un aporte energético, macronutrientes, vitaminas, sales minerales y agua, para mantener un buen estado de salud y rendimiento deportivo [1]. Entre las necesidades nutricionales cabe destacar la ingesta energética, ya que es la que mantiene el peso corporal en los niveles adecuados para el óptimo rendimiento y maximiza los efectos del entrenamiento [2]. La hipertrofia muscular es el incremento de volumen del tejido muscular debido al aumento en el número y tamaño de los elementos contráctiles (filamentos de actina y miosina), lo que se conoce como hipertrofia sarcomérica, o bien debido al aumento de los elementos no contráctiles (colágeno, orgánulos, líquido sarcoplasmático), lo que se conoce como hipertrofia sarcoplasmática [3]. El primer tipo de hipertrofia se acompaña de un aumento en la fuerza manifestada por un músculo, sin embargo, la hipertrofia sarcoplasmática no produce un incremento en la fuerza muscular.

La evidencia científica muestra la importancia de la nutrición en la hipertrofia muscular, especialmente con el objetivo de lograr un balance energético positivo entre la ingesta energética y el consumo de energía, siempre y cuando haya una buena ingesta de proteínas [4], ya que las restricciones calóricas producen un estado de catabolismo muscular [5]. Estudios científicos [6,7] demuestran que la combinación de ejercicio contra resistencia con un sobreaporte energético produce ganancias en la masa corporal magra. Sin embargo, no está clara la cantidad exacta de todos los macronutrientes (hidratos de carbono y grasas). En las proteínas parece haber un cierto consenso, siendo necesarias de 1,6 a $2,0 \mathrm{~g} / \mathrm{kg} /$ día cuando se acompaña de ejercicio contra resistencia, ya que para ganar masa muscular se ha de estar en un equilibrio nitrogenado positivo y para ello la síntesis de proteína debe superar a la degradación $[8,9]$. Sin embargo, en los hidratos de carbono (CHO) y en las grasas no hay 
evidencias que indiquen cuál es la ingesta óptima para el desarrollo de hipertrofia muscular y mejora de la fuerza.

Las dietas más utilizadas para el desarrollo de la masa y la fuerza muscular son la dieta alta en hidratos de carbono y la dieta cetogénica (KD). La dieta alta en hidratos de carbono se caracteriza por una elevada ingesta de calorías aportada por los carbohidratos. En cambio, la dieta cetogénica tiene como objetivo generar una situación de cetosis similar a la del ayuno mediante un aporte insuficiente de alimentos, una restricción de alimentos ricos en glúcidos o bien mediante el consumo de alimentos ricos en grasas o en proteínas.

Existen estudios científicos que comparan la dieta rica en hidratos de carbono con la dieta cetogénica [10-13]. Sin embargo, estos estudios poseen intervenciones muy diferentes, analizando poblaciones de distintos deportes con exigencias metabólicas completamente desiguales. Además, los diferentes sujetos estudiados tampoco han seguido las mismas pautas nutricionales, $\mathrm{y}$ en algunos casos no había una medición exacta de las cantidades alimentarias que debían ingerir. A esto hay que añadir que los estudios mencionados anteriormente no llevan a cabo dietas hipercalóricas junto con entrenamientos de fuerza, por lo que se puede afirmar que no hay evidencias científicas que demuestren qué dieta hipercalórica es mejor para las ganancias hipertróficas y de fuerza en sujetos entrenados que realizan entrenamientos de hipertrofia muscular. Por ello, con el fin de solucionar estas cuestiones se plantea el presente trabajo.

\section{MÉTODOS}

\section{Población de estudio}

Diez varones con más de dos años de experiencia en el entrenamiento sistemático de la fuerza, a los que por lo tanto se les considera sujetos entrenados, se han mostrado voluntarios en la participación de este estudio.

Grupo1: Dieta cetogénica: 5 sujetos.

Grupo 2: Dieta con alto porcentaje de hidratos de carbono: 5 sujetos.

Los criterios de inclusión para seleccionar la población de estudio han sido: pertenecer al sexo masculino, ser jóvenes (edad $24 \pm 6$ ) y tener más de dos años de experiencia en el entrenamiento de hipertrofia. Los participantes fueron excluidos si habían padecido algún tipo de lesión en el último año.

\section{Variables}

Las variables analizadas al inicio y al final del estudio hacen referencia a la composición corporal de los sujetos, calculadas mediante antropometría (peso corporal, porcentaje de grasa, porcentaje de masa muscular, peso graso, peso muscular y peso magro) y respecto a su fuerza, calculada con un test $1 \mathrm{RM}$ en los ejercicios de sentadilla y press banca. 


\section{Dietas}

Los requerimientos calóricos se calcularon de acuerdo con la tasa metabólica basal y el nivel de actividad física diaria [14], a partir de ahí se le elaboró una dieta hipercalórica de $500 \mathrm{kcal} /$ día cetogénica, o rica en hidratos de carbono, según al grupo que perteneciese cada sujeto.

Respecto a las cantidades de macronutrientes, el grupo alto en $\mathrm{HCO}$ consumió $2 \mathrm{~g} / \mathrm{kg} /$ día de proteína [9] y $60 \%$ de hidratos de carbono $[15,16]$, siendo el resto de kcal necesarias aportadas por la grasa. En la dieta cetogénica no se consumieron más de un $5 \%$ de hidratos de carbono al día y $2 \mathrm{~g} / \mathrm{kg}$ ·día de proteína [10]. Además, debido a la imposibilidad de comer ciertos alimentos por su alto contenido en carbohidratos, se ha llevado a cabo una suplementación multivitamínico-mineral $[17,18]$ a fin de evitar deficiencias nutricionales y problemas de acidosis metabólica.

\section{Entrenamiento de fuerza}

Todos los sujetos realizaron el mismo entrenamiento de fuerza, con el fin de reducir las diferencias obtenidas en la composición corporal debidas a este factor. La rutina se basó en un entrenamiento de cuerpo dividido, es decir, en cada entrenamiento se realizó tren superior o tren inferior [19], pasando 72 horas de recuperación entre los mismos grupos musculares [20]. Respecto al volumen del entrenamiento, se llevó a cabo volúmenes de 6 series por grupo muscular y sesión y de 42 a 66 repeticiones, con tiempos de recuperación entre series de 2 minutos [21-23]. En cuanto a la carga, se entrenó a niveles medios, 6-12 RM [3] y además todas las series se realizaron al fallo muscular [24].

\section{Análisis estadístico}

El análisis estadístico se realizó mediante el software informático SPSS. Se calculó la normalidad de la muestra y a continuación se llevó a cabo la prueba T. Las diferencias fueron consideradas significativas cuando $\mathrm{p}<0,05$.

\section{RESULTADOS}

Ambas dietas produjeron un aumento significativo en el porcentaje de masa muscular y en el $1 \mathrm{RM}$ de press banca y sentadilla (figuras 2 y 4), siendo este aumento más destacado en la dieta rica en carbohidratos. La dieta alta en hidratos de carbono aumentó significativamente en 1,25 \% la masa muscular (figura 2A); 11,1 la 1RM press banca y 31,5 la 1RM sentadilla (figura 2B). 


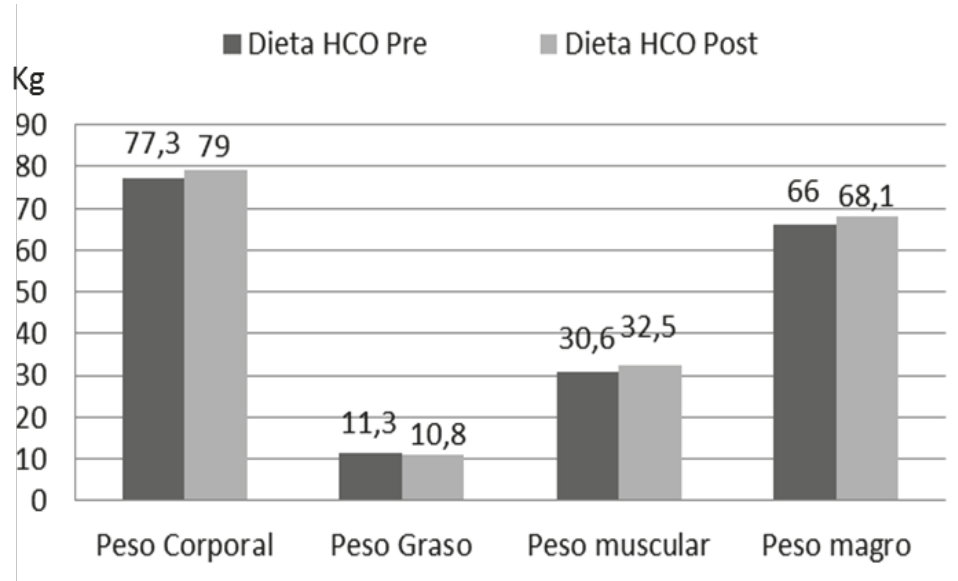

Fig. 1. Variación de las medias del peso corporal, peso graso, peso muscular y peso magro de los sujetos antes (pre) y después (post) de someterse a la dieta alta en hidratos de carbono (HCO)
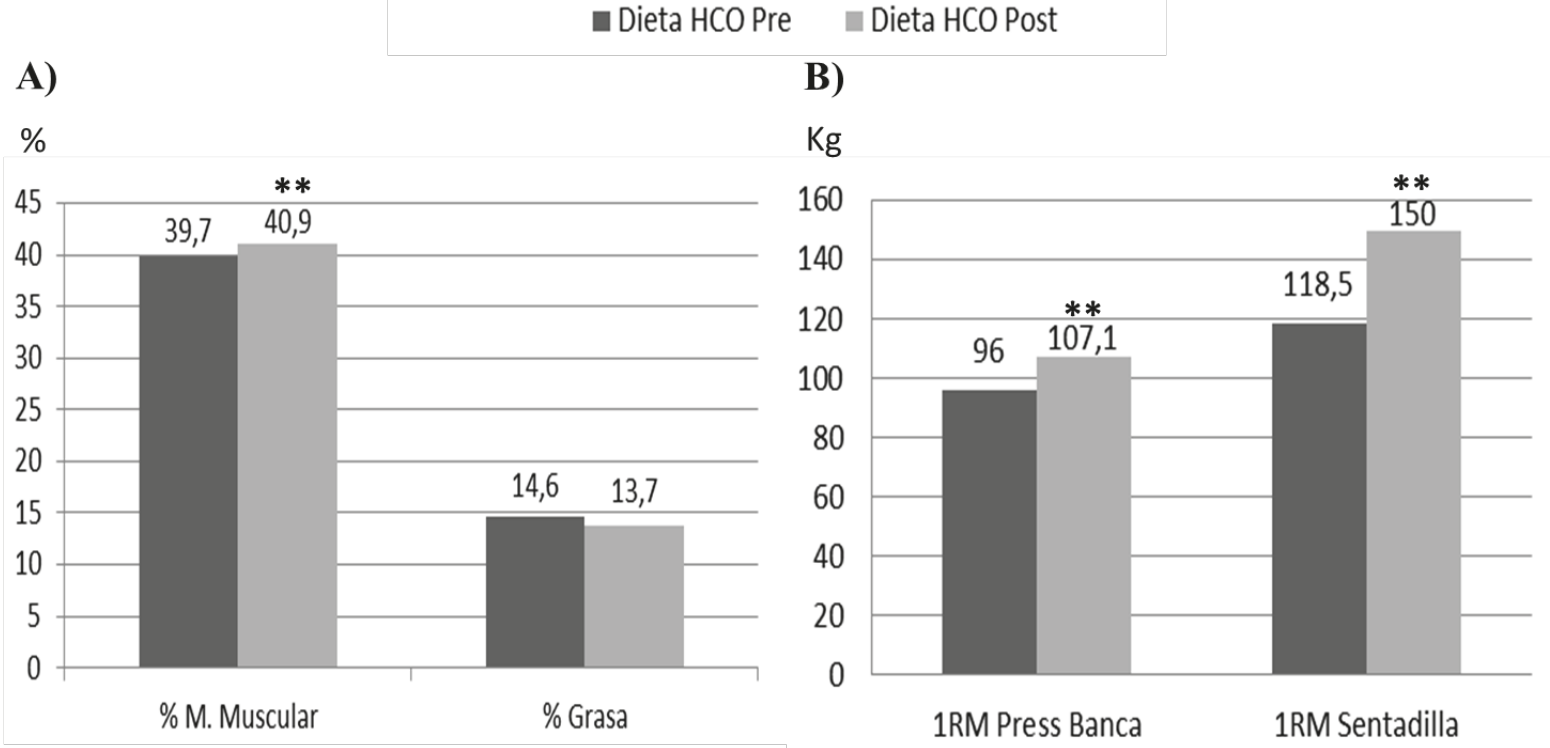

Fig. 2. Variación de las medias de A) porcentaje de masa muscular y de grasa; B) 1RM press banca y 1RM sentadilla de los sujetos antes (pre) y después (post) de someterse a la dieta alta en hidratos de carbono (HCO). Las diferencias significativas se muestran como $* \mathrm{p}<0,05 ; * \mathrm{p}<0,01$; pre $v s$. post.

La dieta cetogénica aumentó significativamente en 1,96\% la masa muscular (figura 4A); 7,5 la 1RM press banca y 16,5 la 1RM sentadilla (figura 4B). Además, la dieta cetogénica destacó por la disminución significativa de $1,79 \%$ de grasa (figura $4 \mathrm{~A}$ ) y de 1,22 en el peso graso (figura 3 ). 


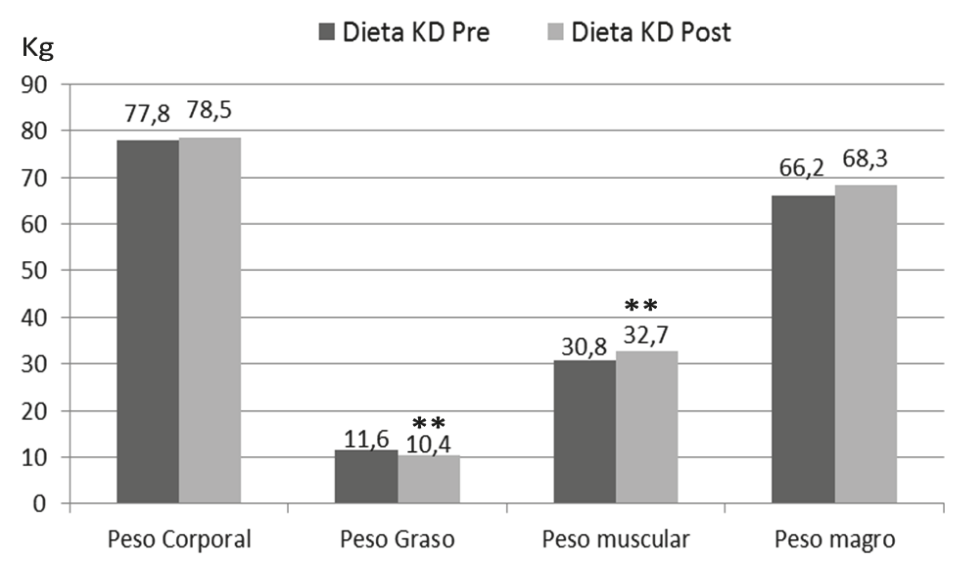

Fig. 3. Variación de las medias del peso corporal, peso graso, peso muscular y peso magro de los sujetos antes (pre) y después (post) de someterse a la dieta cetogénica (KD). Las diferencias significativas se muestran como $* \mathrm{p}<0,05 ; * \mathrm{p}<0,01$; pre $v s$. post.

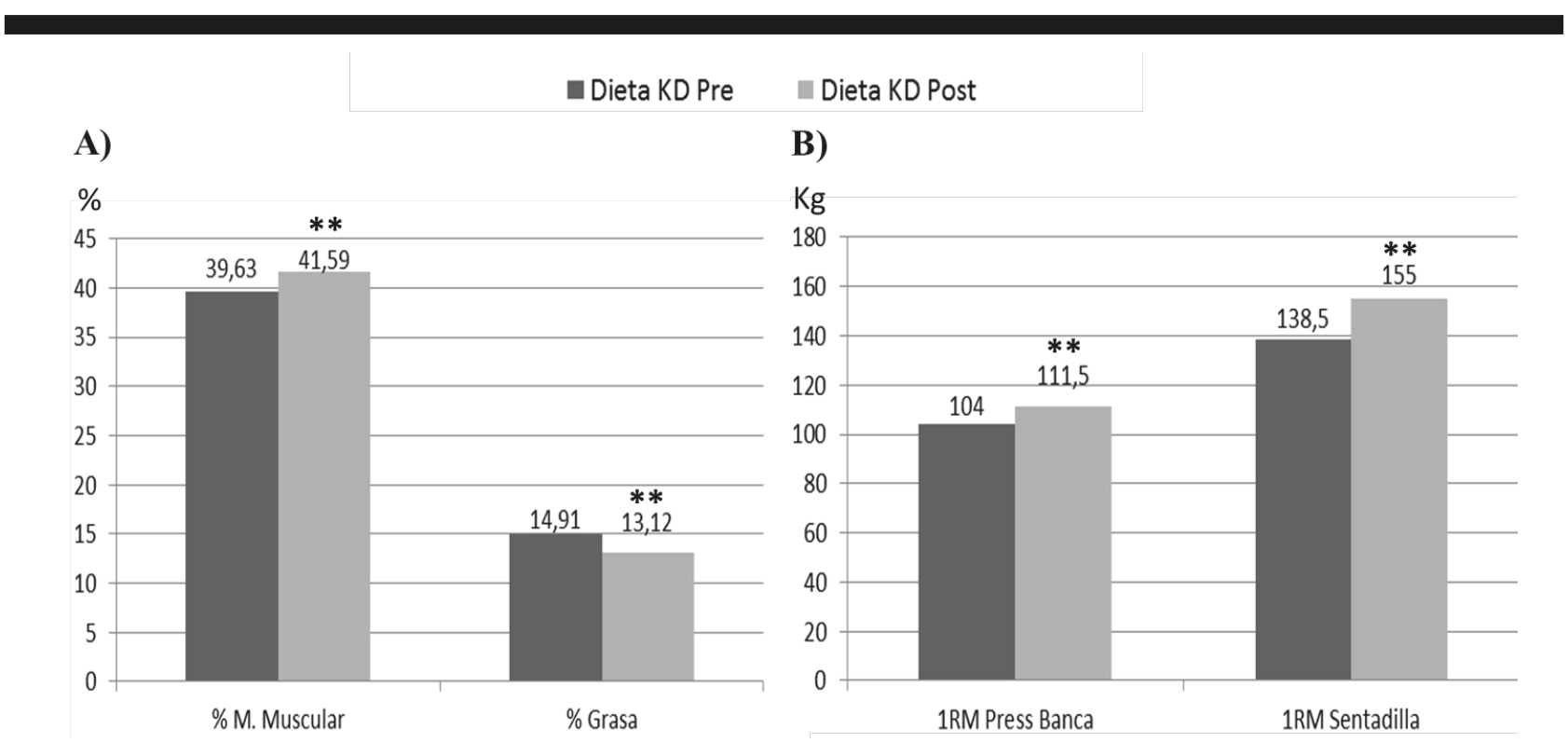

Fig. 4. Variación de las medias de A) porcentaje de masa muscular y de grasa; B) 1RM press banca y 1RM sentadilla, de los sujetos antes (pre) y después (post) de someterse a la dieta cetogénica (KD). Las diferencias significativas se muestran como $* \mathrm{p}<0,05 ; * * \mathrm{p}<0,01 ;$ pre $v s$. post.

\section{DISCUSIÓN}

Como ya se comentó anteriormente, la hipertrofia muscular y la dieta cetogénica son temas muy demandados en la sociedad actual y sin embargo no poseen demasiada evidencia científica conjuntamente. Tras observar la importancia de la nutrición en el desarrollo de la masa magra, surgen dudas 
como qué cantidad de calorías son necesarias, qué tipo de dietas son las más eficaces o qué cantidades de macronutrientes se deberían ingerir, siendo estas dos últimas no resueltas por la comunidad científica.

Algunos autores [25] proponen recomendaciones de 4 a $7 \mathrm{~g} / \mathrm{kg} /$ día de hidratos de carbono para atletas de fuerza, aunque sin llegar a evidencias concluyentes de ser necesarias altas dosis. La dieta cetogénica ha sido muy investigada en los últimos años como dieta para la reducción de peso corporal y del riesgo cardiovascular [26-28], sin embargo, su efecto en el rendimiento deportivo como dieta hipercalórica con entrenamientos de fuerza apenas ha sido investigado. Existen investigaciones que comparan dietas altas en $\mathrm{HCO}$ respecto a las cetogénicas con otro tipo de intervenciones [10-13].

Por ello, este estudio aporta una referencia en cuanto a cantidades de macronutrientes y tipo de entrenamiento a seguir para conseguir un aumento de la masa muscular, ya que los dos grupos de sujetos tienen mejoras significativas en algunas variables de la medición inicial a la final tras las 8 semanas de intervención. En el grupo con dieta alta en $\mathrm{HCO}$, han obtenido mejoras en todas las variables, peso corporal, porcentaje de masa muscular, porcentaje de grasa, peso graso, peso muscular, peso magro y 1RM en press banca y sentadilla, sin embargo, solo han sido significativas las mejoras en el porcentaje de masa muscular y en el 1RM en press banca y sentadilla. Respecto al grupo con dieta cetogénica, todas las variables poseen diferencias significativas salvo el peso corporal y el peso magro, que aunque han aumentado no llega a ser significativo; esto es debido principalmente a la pérdida de grasa, la cual baja el peso corporal total.

\section{CONCLUSIONES}

Tanto la dieta alta en hidratos de carbono como la dieta cetogénica estarían indicadas para personas que quieran mejorar su hipertrofia y fuerza llevando a cabo entrenamiento de hipertrofia. Sin embargo, si el objetivo tiene una finalidad más estética, la dieta cetogénica podría aportar algo más de resultados, mientras que si hay un objetivo más de mejora de la fuerza, sería más recomendable una dieta rica en hidratos de carbono.

\section{AGRADECIMIENTOS}

Este trabajo no hubiera sido posible sin la contribución de las personas que se han prestado como voluntarias a participar en la intervención y se han comprometido a llevar a cabo un entrenamiento y dietas estrictas, sin las cuales este proyecto no se hubiera podido realizar. Gracias también a los laboratorios Nutergia por el suministro de los productos multivitamínicos y minerales.

\section{REFERENCIAS BIBLIOGRÁFICAS}

[1] Sanz JMM, Otegui AU, Ayuso JM. Necesidades energéticas, hídricas y nutricionales en el deporte. Eur J Hum Mov. 2013;30:37-52. 
[2] González-Gross M, Gutiérrez A, Mesa JL, Ruiz-Ruiz J, Castillo MJ. Nutrition in the sport practice: adaptation of the food guide pyramid to the characteristics of athletes diet. Arch Latinoam Nutr. 2001 Dec.51;(4):321-31. PMID 12012556.

[3] Schoenfeld BJ. The mechanisms of muscle hypertrophy and their application to resistance training. J Strength Cond Res. 2010 Oct;24(10):2857-72. DOI: 10.1519/JSC.0b013e3181e840f3.

[4] Churchward-Venne TA, Murphy CH, Longland TM, Phillips SM. Role of protein and amino acids in promoting lean mass accretion with resistance exercise and attenuating lean mass loss during energy deficit in humans. Amino Acids. 2013 Aug;45(2):231-40. DOI: 10.1007/s00726013-1506-0.

[5] McIver CM, Wycherley TP, Clifton PM. MTOR signaling and ubiquitin-proteosome gene expression in the preservation of fat free mass following high protein, calorie restricted weight loss. Nutr Metab. 2012 Sep 14;9(1):83. DOI: 10.1186/1743-7075-9-83.

[6] Garthe I, Raastad T, Refsnes PE, Sundgot-Borgen J. Effect of nutritional intervention on body composition and performance in elite athletes. Eur J Sport Sci. 2013;13(3):295-303. DOI: 10.1080/17461391.2011.643923.

[7] Rozenek R, Ward P, Long S, Garhammer J. Effects of high-calorie supplements on body composition and muscular strength following resistance training. J Sports Med Phys Fitness. 2002 Sep;42(3):340-7. PMID: 12094125.

[8] Lemon PW, Tarnopolsky MA, MacDougall JD, Atkinson SA. Protein requirements and muscle mass/strength changes during intensive training in novice bodybuilders. J Appl Physiol Bethesda Md 1985. 1992 Aug;73(2):767-75. DOI: 10.1152/jappl.1992.73.2.767.

[9] Campbell B, Kreider RB, Ziegenfuss T, La Bounty P, Roberts M, Burke D, et al. International Society of Sports Nutrition position stand: protein and exercise. J Int Soc Sports Nutr. 2007 Sep 26;4(1):8. DOI: 10.1186/1550-2783-4-8.

[10] Paoli A, Grimaldi K, D’Agostino D, Cenci L, Moro T, Bianco A, et al. Ketogenic diet does not affect strength performance in elite artistic gymnasts. J Int Soc Sports Nutr. 2012 Dec;9(1):34. DOI: $10.1186 / 1550-2783-9-34$.

[11] Wilson JM, Lowery RP, Roberts MD, Sharp MH, Joy JM, Shields KA, et al. The effects of ketogenic dieting on body composition, strength, power, and hormonal profiles in resistance training males. J Strength Cond Res. 2017 Apr 7; DOI: 10.1519/JSC.0000000000001935.

[12] McSwiney FT, Wardrop B, Hyde PN, Lafountain RA, Volek JS, Doyle L. Keto-adaptation enhances exercise performance and body composition responses to training in endurance athletes. Metabolism. 2018 Apr 1;81:25-34. DOI: 10.1016/j.metabol.2017.10.010.

[13] Kephart WC, Pledge CD, Roberson PA, Mumford PW, Romero MA, Mobley CB, et al. The three-month effects of a ketogenic diet on body composition, blood parameters, and performance metrics in crossfit trainees: A Pilot Study. Sports (Basel). 2018 Mar;6(1):1. DOI: 10.3390/ sports6010001.

[14] Tagliabue A, Bertoli S, Trentani C, Borrelli P, Veggiotti P. Effects of the ketogenic diet on nutritional status, resting energy expenditure, and substrate oxidation in patients with medically refractory epilepsy: A 6-month prospective observational study. Clin Nutr. 2012 Apr 1;31(2):2469.DOI: 10.1016/j.clnu.2011.09.012. 
[15] Thomas DT, Erdman KA, Burke LM. American college of sports medicine joint position statement. Nutrition and athletic performance. Med Sci Sports Exerc. 2016 Mar;48(3):543-68. DOI: 10.1249/MSS.0000000000000852.

[16] Lane AR, Duke JW, Hackney AC. Influence of dietary carbohydrate intake on the free testosterone: cortisol ratio responses to short-term intensive exercise training. Eur J Appl Physiol. 2010 Apr;108(6):1125-31. DOI: 10.1007/s00421-009-1220-5.

[17] Phinney SD, Horton ES, Sims EAH, Hanson JS, Danforth E, Lagrange BM. Capacity for moderate exercise in obese subjects after adaptation to a hypocaloric, ketogenic diet. J Clin Invest. 1980 Nov;66(5):1152-61. DOI: 10.1172/JCI109945

[18] Zupec-Kania B, Zupanc ML. Long-term management of the ketogenic diet: seizure monitoring, nutrition, and supplementation. Epilepsia. 2008 Nov;49 Suppl 8:23-6. DOI: 10.1111/j.15281167.2008.01827.x.

[19] Helms ER, Fitschen PJ, Aragon AA, Cronin J, Schoenfeld BJ. Recommendations for natural bodybuilding contest preparation: resistance and cardiovascular training. J Sports Med Phys Fitness. 2015 Mar;55(3):164-78. PMID: 24998610

[20] MacDougall JD, Gibala MJ, Tarnopolsky MA, MacDonald JR, Interisano SA, Yarasheski KE. The time course for elevated muscle protein synthesis following heavy resistance exercise. Can J Appl Physiol Rev Can Physiol Appl. 1995 Dec;20(4):480-6. PMID: 8563679.

[21] Buresh R, Berg K, French J. The effect of resistive exercise rest interval on hormonal response, strength, and hypertrophy with training. J Strength Cond Res. 2009 Jan;23(1):62-71. DOI: 10.1519/JSC.0b013e318185f14a.

[22] De Souza TP, Fleck SJ, Simão R, Dubas JP, Pereira B, de Brito Pacheco EM, et al. Comparison between constant and decreasing rest intervals: influence on maximal strength and hypertrophy. J Strength Cond Res. 2010 Jul;24(7):1843-50. DOI: 10.1519/JSC.0b013e3181ddae4a.

[23] Schoenfeld BJ, Pope ZK, Benik FM, Hester GM, Sellers J, Nooner JL, et al. Longer interset rest periods enhance muscle strength and hypertrophy in resistance-trained men. J Strength Cond Res. 2016 Jul;30(7):1805-12. DOI: 10.1519/JSC.0000000000001272.

[24] Chulvi-Medrano I, García-Massó X, Colado JC, Pablos C, de Moraes JA, Fuster MA. Deadlift muscle force and activation under stable and unstable conditions. J Strength Cond Res. 2010 Oct;24(10):2723-30. DOI: 10.1519/JSC.0b013e3181f0a8b9.

[25] Slater G, Phillips SM. Nutrition guidelines for strength sports: sprinting, weightlifting, throwing events, and bodybuilding. J Sports Sci. 2011; 29 Suppl 1, S67-77. DOI: 10.1080/02640414.2011.574722.

[26] Paoli A. Ketogenic diet for obesity: friend or foe? Int J Environ Res Public Health. 2014 Feb 19;11(2),2092-2107. DOI: 10.3390/ijerph110202092.

[27] Dashti HM, Mathew TC, Hussein T, Asfar SK, Behbahani A, Khoursheed MA, et al. Longterm effects of a ketogenic diet in obese patients. Exp Clin Cardiol. 2004;9(3), 200-5. PMID: 19641727.

[28] Kosinski C, Jornayvaz FR. Effects of ketogenic diets on cardiovascular risk factors: evidence from animal and human studies. Nutrients. 2017 May 19;9(5). DOI: 10.3390/nu9050517. 
